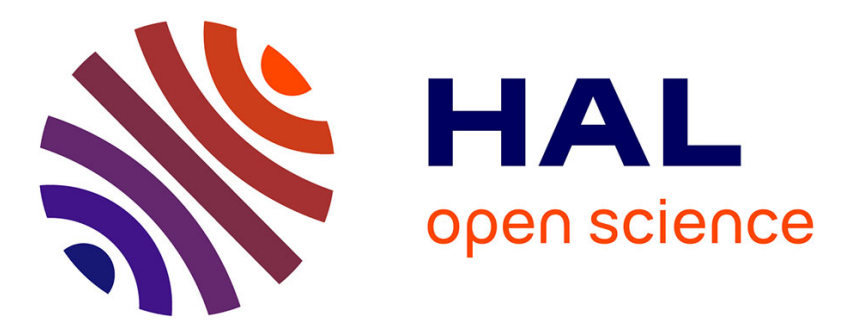

\title{
Subwavelength imaging of light confinement in high-Q/small-V photonic crystal nanocavity
}

\author{
L. Lalouat, B. Cluzel, F. de Fornel, P. Velha, Philippe Lalanne, D. Peyrade,
} E. Picard, T. Charvolin, E. Hadji

\section{- To cite this version:}

L. Lalouat, B. Cluzel, F. de Fornel, P. Velha, Philippe Lalanne, et al.. Subwavelength imaging of light confinement in high-Q/small-V photonic crystal nanocavity. Applied Physics Letters, 2008, 92 (11), pp.111111. 10.1063/1.2890051 . hal-00394794

\section{HAL Id: hal-00394794 \\ https://hal.science/hal-00394794}

Submitted on 11 Apr 2016

HAL is a multi-disciplinary open access archive for the deposit and dissemination of scientific research documents, whether they are published or not. The documents may come from teaching and research institutions in France or abroad, or from public or private research centers.
L'archive ouverte pluridisciplinaire HAL, est destinée au dépôt et à la diffusion de documents scientifiques de niveau recherche, publiés ou non, émanant des établissements d'enseignement et de recherche français ou étrangers, des laboratoires publics ou privés. 


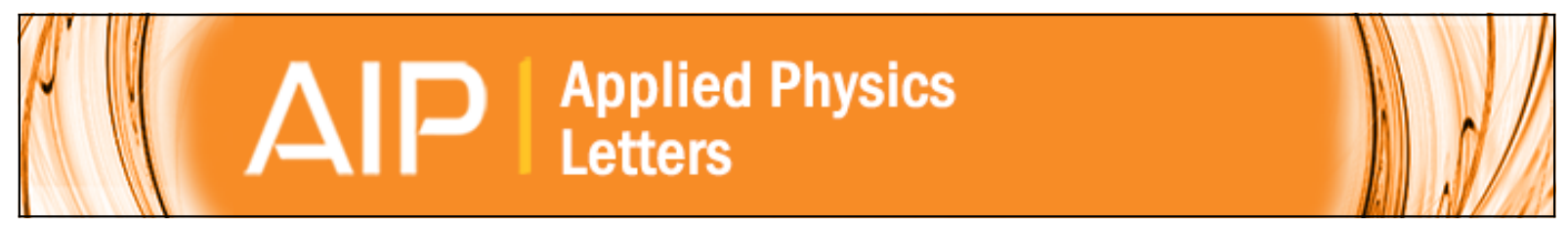

\section{Subwavelength imaging of light confinement in high- Q /small- V photonic crystal nanocavity}

Loic Lalouat, Benoit Cluzel, Frédérique de Fornel, Philippe Velha, Philippe Lalanne, David Peyrade, Emmanuel Picard, Thomas Charvolin, and Emmanuel Hadji

Citation: Applied Physics Letters 92, 111111 (2008); doi: 10.1063/1.2890051

View online: http://dx.doi.org/10.1063/1.2890051

View Table of Contents: http://scitation.aip.org/content/aip/journal/apl/92/11?ver=pdfcov

Published by the AIP Publishing

\section{Articles you may be interested in}

Nonlinear switching dynamics in a photonic-crystal nanocavity

Appl. Phys. Lett. 105, 071112 (2014); 10.1063/1.4893984

Addressable subwavelength grids of confined light in a multislotted nanoresonator Appl. Phys. Lett. 98, 081101 (2011); 10.1063/1.3555489

Digital resonance tuning of high- $\mathrm{Q} / \mathrm{V} \mathrm{m}$ silicon photonic crystal nanocavities by atomic layer deposition Appl. Phys. Lett. 91, 161114 (2007); 10.1063/1.2800312

Subwavelength imaging of field confinement in a waveguide-integrated photonic crystal cavity

J. Appl. Phys. 98, 086109 (2005); 10.1063/1.2115090

Near-field scanning optical microscopy of photonic crystal nanocavities

Appl. Phys. Lett. 82, 1676 (2003); 10.1063/1.1559646

\section{AIP $\mid$ APL Photonics}

APL Photonics is pleased to announce Benjamin Eggleton as its Editor-in-Chief

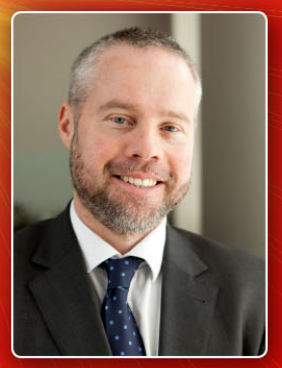




\title{
Subwavelength imaging of light confinement in high- $Q /$ small- $V$ photonic crystal nanocavity
}

\author{
Loic Lalouat, ${ }^{1}$ Benoit Cluzel, ${ }^{1, a)}$ Frédérique de Fornel, ${ }^{1}$ Philippe Velha, ${ }^{2}$ Philippe Lalanne, ${ }^{2}$ \\ David Peyrade, ${ }^{3}$ Emmanuel Picard, ${ }^{4}$ Thomas Charvolin, ${ }^{4}$ and Emmanuel Hadji ${ }^{4}$ \\ ${ }^{1}$ Groupe d'Optique de Champ Proche, Institut Carnot de Bourgogne UMR 5209 CNRS-9 av. A. Savary, \\ 21078 DIJON, France \\ ${ }^{2}$ Laboratoire Charles Fabry de l'Institut d'Optique, CNRS, Université Paris-Sud, Campus Polytechnique, \\ RD128, 91127 Palaiseau, France \\ ${ }^{3}$ Laboratoire des Technologies de la Microélectronique, CNRS, 38054 GRENOBLE, France \\ ${ }^{4}$ Laboratoire Silicium Nanoélectronique Photonique et Structures, DRFMC/SP2M,CEA Grenoble, 17 rue des \\ Martyrs, 38054 GRENOBLE, France
}

(Received 11 December 2007; accepted 8 February 2008; published online 20 March 2008)

\begin{abstract}
The optical near field of a high- $Q$ and ultrasmall volume photonic crystal nanocavity is visualized with a subwavelength resolution by using a scanning near-field optical microscope (SNOM) operating at the same time in collection-scanning mode and in interaction-scanning mode. It is shown that the nanocavity resonant mode is selectively visualized by using the SNOM interaction-scanning mode while the whole electromagnetic field surrounding the nanocavity is probed using the SNOM collection-scanning mode. The different optical near-field images are compared in light of a three-dimensional numerical analysis and we demonstrate an unexpected mode coupling at the cavity resonance. (C) 2008 American Institute of Physics. [DOI: 10.1063/1.2890051]
\end{abstract}

Semiconductor photonic crystal resonators have attracted much attention over the recent years. Their potential for high quality (high- $Q$ ) factor in small volume $(V)$ cavities opens innovative ways to control light, including the stopping and the trapping light, ${ }^{1}$ quantum information processing, ${ }^{2,3}$ and the enhancement of light-matter interactions. ${ }^{4}$ This potential is expected to lead to a wide range of applications for integrated on-chip photonics. ${ }^{5-7}$

The methods most commonly used for examining the properties of such nanocavities are optical far-field techniques, which typically consist in analyzing (spectrally or temporally) the photons radiated into the far field. However, these methods provide only indirect insight in the lightmatter interactions occurring in the cavity optical near-field, which are at the origin of the remarkable properties of these cavities. As a result, scanning near-field optical microscopy techniques (SNOM) are highly relevant to directly probe such nanostructures. ${ }^{8}$ As reported in Refs. 9-11, the SNOM technique is a powerful method for localizing, both spatially and spectrally, the electromagnetic field confinement inside photonic crystal cavities. However, only low- $Q$ cavities have been studied so far and the very first attempts at probing small volume cavities have shown that the near-field probes [SNOM probes in Ref. 12 and atomic force microscopy (AFM) probes in Refs. 13 and 14] strongly alter-or even completely disrupt in the case of AFM probes-the light confinement mechanism inside the cavity. For these reasons, probing with a high resolution the optical near-field of the light confined inside a high- $Q /$ small- $V$ nanocavity is a challenging task.

In this study, we face this challenge and achieve experimentally high resolution SNOM pictures of the light confined inside a nanocavity exhibiting a $Q$-factor of 41000 (40 times higher than our previous works in Ref. 12 and 80 times

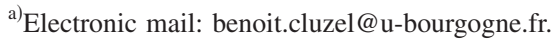

higher than in Refs. 13 and 14) and a modal volume of 5.5.10 $0^{-14} \mathrm{~cm}^{3}$ (almost 15 times lower than in Ref. 13). The near-field images reported here are simultaneously recorded using the recently proposed SNOM interaction-scanning mode, ${ }^{12,13,15}$ and the classical SNOM collection-scanning mode. ${ }^{8-11}$ The differences between these two imaging methods are discussed in light of the recorded images by comparing them with three-dimensional (3D) calculations of the cavity mode.

The nanocavity considered in this work is a FabryPerot-like resonator integrated on a ridge waveguide, designed in such a way to suppress the radiation losses at the mirror termination. ${ }^{16}$ Similar structures have been recently investigated by other group. ${ }^{17}$ The cavity is fabricated by electron beam lithography and reactive ion etching on a silicon-on-insulator substrate. As shown in Fig. 1, for a transverse electrical (TE with the H-field is normal to the substrate plane) polarization of the light, the nanocavity ${ }^{18}$ exhibits a single resonance at telecommunication wavelength $\left(\lambda_{0}\right.$ $\sim 1558 \mathrm{~nm}$ ) with a $Q$-factor of 41000 .

The sample is then mounted on a SNOM coupled to a spectrally resolved optical bench. A TE polarized tunable laser source is coupled to the access waveguide by using a lensed fiber (Lovalite ${ }^{\circledR}$ Photonics Tip). The light transmitted through the cavity is collected using an achromatic metallic mirror objective and detected using an InGaAs photodiode. By accurately maintaining the optical alignments, an ultrasmall homemade near-field probe consisting in a chemically etched silica fiber with a $20 \mathrm{~nm}$ width apex is scanned at a $4 \mathrm{~nm}$ height above the cavity surface, while the probesurface distance is controlled thanks to a shear-force feedback. ${ }^{19}$

As expected for a nanometric-sized dielectric probe, ${ }^{12,20}$ the cavity resonance wavelength is slightly redshifted when the probe is introduced inside the cavity optical near-field. Remarkably, due to the extremely small size of the probe and 


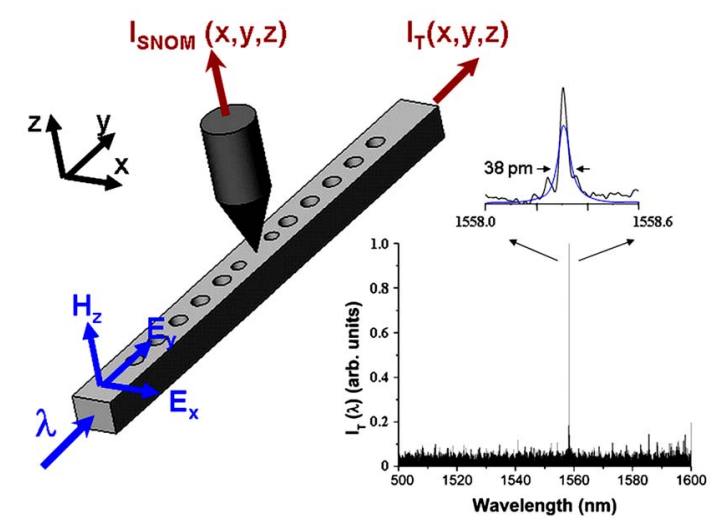

(a)

FIG. 1. (Color online) (a) Schematic of the experiment. A TE polarized tunable laser is coupled to the nanocavity. As the near-field probe is scanned over the cavity surface, the light collected by the probe $\left(I_{\mathrm{SNOM}}\right)$ and the light transmitted through the cavity $\left(I_{T}\right)$ are collected at the same time. The first operating method, $I_{\mathrm{SNOM}}(x, y, z)$, corresponds the classical SNOM collection-scanning mode, while the second one, $I_{T}(x, y, z)$, is the interaction-scanning mode. (b) Typical nanocavity transmittance spectra. The experimental spectrum exhibits a Lorentzian-shaped peak at resonance.

to its relative small refractive index, the resonance wavelength is redshifted $(\delta \lambda=0.2 \mathrm{~nm})$, while the cavity $Q$-factor is only lowered by a factor 2. Consequently, the near-field probe used here operates in a perturbation regime weak enough to avoid a drastic alteration of the light confinement inside the cavity. ${ }^{12,20}$

For SNOM measurements, we then develop an experimental setup allowing the simultaneous recording of the near-field maps of the nanocavity in collection-scanning mode $^{8-11}$ and interaction-scanning mode. ${ }^{12,13,21}$ The first method is similar to the one used in classical apertureSNOM measurements, and the probe is used to locally collect the electromagnetic field above the cavity $\left[I_{\mathrm{SNOM}}(x, y, z)\right.$ in Fig. 1]. The second operating mode is based on the cavity transmittance-variations $\left[I_{T}(x, y, z)\right.$ in Fig. 1], which depend on the probe position above the nanocavity. Figure 2 shows typical near-field images recorded using the two operating modes at the cavity resonance wavelength modified by the presence of the probe $\left(\lambda_{0}+\delta \lambda\right)$. In addition, for the purpose of providing an insight in the spatial localization of the nanocavity on the optical near-field images, we present the shearforce feed-back signal recorded during the optical scans in Fig. 2(b). Figure 2(a) additionally shows the scanning electron microscopy (SEM) image of the cavity.

Let us first analyze the image recorded in interactionscanning mode. As explained in Refs. 12 and 21, the nearfield map is proportional to the resonant electric field distribution inside the cavity. Thus, the positions of hot (or dark) spots are entirely determined by the position of the nodes (or antinodes) of the total electric field intensity. To allow for a direct comparison, we plotted in Fig. 3(a) the total electric field intensity, i.e., $\left\|\vec{E}_{x}\right\|^{2}+\left\|\vec{E}_{y}\right\|^{2}+\left\|\vec{E}_{z}\right\|^{2}$, computed at a $4 \mathrm{~nm}$ height above the cavity surface by using a $3 \mathrm{D}$ fully vectorial frequency-domain modal method relying on Fourier expansion techniques. ${ }^{22}$ A rapid glance at the two images reveals strong similarities between the numerical one in Fig. 3(a) and the experimental one in Fig. 2(c).

In contrast to the interaction-scanning mode which detects photons outcoupled from the cavity, the collectionscanning mode is directly related to the photons surrounding
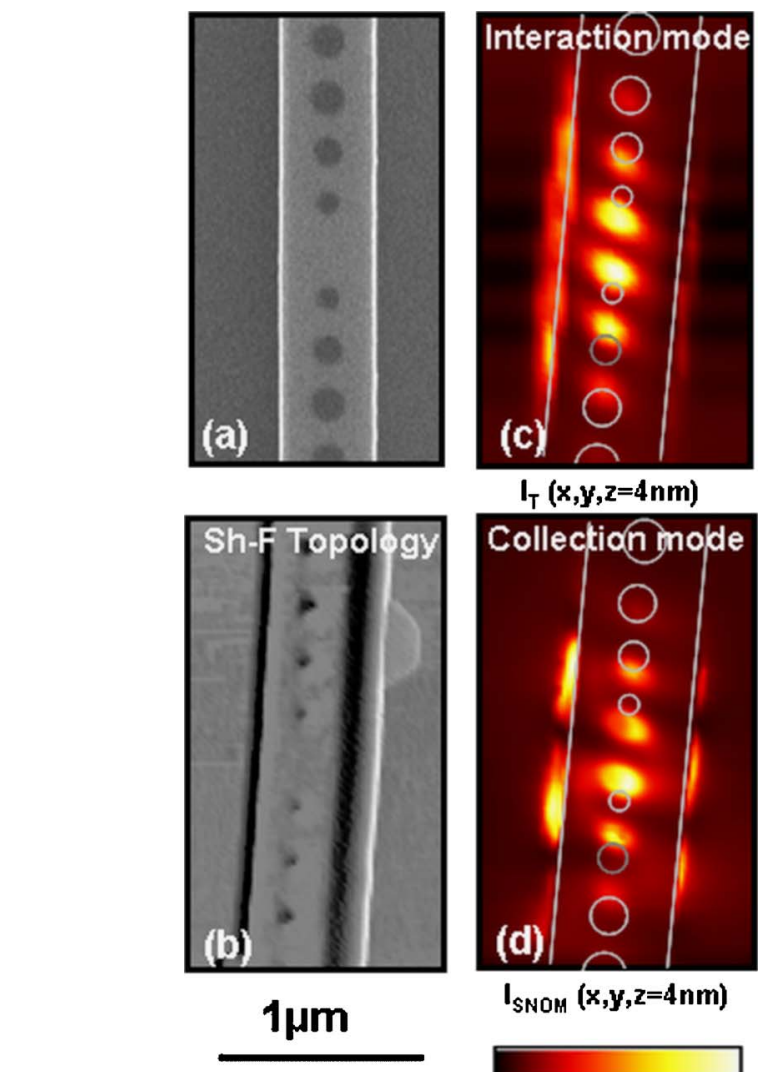

$\mathrm{I}_{\mathrm{T}}(\mathrm{x}, \mathrm{y}, \mathrm{z}=\mathbf{4 n m})$
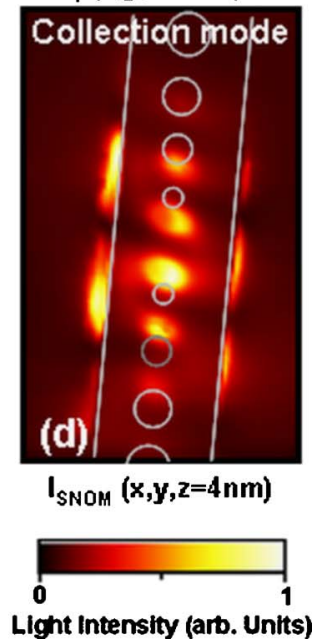

FIG. 2. (Color online) (a) SEM picture of cavity. (b) Shear-force feedback signal recorded during the probe scan above the structure. (c) Interactionscanning mode map and (d) Collection-scanning mode map of the light confined at the cavity resonance.

the cavity optical near-field. As will be shown hereafter, the two imaging modes are complementary, and both of them must be taken into account in order to achieve a stringent analysis of the whole electromagnetic field surrounding the nanocavity at resonance.

A comparison between the maps recorded using the two operating modes [Figs. 2(c) and 2(d)] unambiguously shows their similarities and differences. The cavity resonant mode signature is clearly present in each of the two mapping modes, with hot and dark spots appearing at the position of the nodes and antinodes of the electric field. However, by contrast with the interaction-mode image, the collection mode image additionally exhibits a phase-shifted beating of

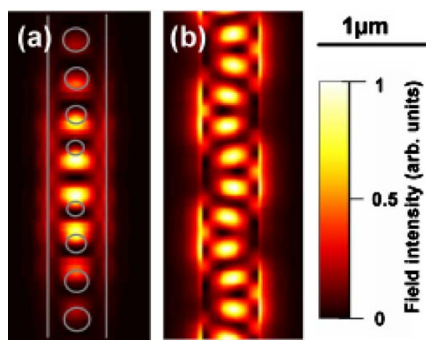

FIG. 3. (Color online) (a) 3D calculations of the resonant mode intensity at $4 \mathrm{~nm}$ above the nanocavity: $\|\vec{E}\|^{2}=\left\|\vec{E}_{x}\right\|^{2}+\left\|\vec{E}_{y}\right\|^{2}+\left\|\vec{E}_{z}\right\|^{2}$. (b) Computed electric field intensity distribution above a SOI waveguide resulting from the propagation of the fundamental mode and the first order mode. The figure illustrates the ohase-shifted beating pattern on the edge of the waveguide that can be observed in the SNOM collection mode measurements. 
alternate hot and dark spots on the edge of the waveguide. We note that the contrast is maximal in the central part of the cavity and that it rapidly vanishes as one enters the mirrors. Additionally, we also note that the characteristic beating length is $\sim 600 \mathrm{~nm}$, which surprisingly corresponds to twice the separation distance between the standing wave antinodes (or nodes) in the middle of the waveguide.

As the alternation of the hot and dark spots along the two edges of the waveguide does not respect its transversal symmetry, this implies that at least two modes with an opposite parity are coexisting inside the structure, leading to a phase-shifted beating pattern on the SNOM collection mode picture. ${ }^{23}$ Such a phenomenon is generally attributed to the summation of the field amplitudes of the modes inside the SNOM probe. Moreover, as the silicon waveguide is bimodal, we believe that the observed beating results from the co-existence of the fundamental mode (with an even parity) bouncing between the two mirrors and the first order mode (with an odd parity) propagating along the waveguide. We computed the beating pattern expected for these two modes in the case of the silicon waveguide. The result is plotted in Fig. 3(b). It clearly exhibits a beating pattern similar to that one observed experimentally with a characteristic beating length close to the measured value.

Consequently, we believe that the SNOM collection mode picture results from the competition of the cavity resonant mode electric field distribution and of the beating pattern. At last, we underline that the beating is not observed elsewhere along the ridge waveguide except in the vicinity of the cavity resonant mode. Thus, we believe that the first order mode is not propagating along the whole waveguide but is rather excited inside the cavity by the resonant fundamental mode itself. The origin of this coupling, which is obviously indirect since the two modes are orthogonal, is currently under investigation.

In conclusion, we have presented optical near-field maps of the light confined within a high- $Q /$ small- $V$ photonic crystal nanocavity recorded in interaction-scanning mode and in collection-scanning mode. The differences between these two imaging methods have been analyzed and the comparison between the different pictures has been found to be supported by three dimensional field distribution calculations. The interaction-scanning mode is found to be selectively sensitive to the cavity resonant mode field distribution while the collection-scanning mode detects the whole electromagnetic field surrounding the nanocavity. In addition, the comparison between the two imaging methods has revealed unexpected mode coupling at the cavity resonance. Finally, since we were able to overcome the challenge of directly probing the optical near-field of the nanocavity without drastically altering its light confinement, we believe this study to a step towards the in situ mechanical nanomanipulation of confined electromagnetic fields ${ }^{24}$ for the control of the quantum nature of strongly coupled cavity-atom systems of solidstate physics.

This work has received financial support from the French ministry in charge of research in the context of the
ACI NR 63 CHABIP. This work is also partly supported by the French national program PNANO from the "Agence Nationale de la Recherche" under the MIRAMAN contract.

${ }^{1}$ T. Tanabe, M. Notomi, E. Kuramochi, A. Shinya1, and H. Taniyama, Nat. Photonics 1, 49 (2007).

${ }^{2}$ K. Hennessy, A. Badolato, M. Winger, D. Gerace, M. Atatüre, S. Gulde, S. Fält, E. L. Hu, and A. Imamoglu, Nature (London) 445, 896 (2007).

${ }^{3}$ T. Yoshie, A. Scherer, J. Hendrickson, G. Khitrova, H. M. Gibbs, G. Rupper, C. Ell, O. B. Shchekin, and D. G. Deppe, Nature (London) 432, 200 (2004)

${ }^{4}$ M. Soljacic and J. D. Joannopoulos, Nat. Mater. 3, 211 (2004).

${ }^{5}$ For microlasers see, for example, O. Painter, R. K. Lee, A. Scherer, A. Yariv, J. D. O'Brien, P. D. Dapkus, and I. Kim, Science 284, 1819 (1999); H.-G. Park, S.-H. Kim, S.-H. Kwon, Y.-G. Ju, J.-K. Yang, J.-H. Baek, S.-B. Kim, and Y.-H. Lee, ibid. 305, 1444 (2004).

${ }^{6}$ For sensing see, for example, M. Loncar, A. Scherer, and Y. Qiu, Appl. Phys. Lett. 82, 4648 (2003); B. Schmidt, V. Almeida, C. Manolatou, S. Preble, and M. Lipson, ibid. 85, 4854 (2004).

${ }^{7}$ For switching see, for example, I. Fushman, E. Waks, D. Englund, N. Stoltz, P. Petroff, and J. Vučković, Appl. Phys. Lett. 90, 091118 (2007); B. Schmidt, Q. Xu, J. Shakya, S. Manipatruni, and M. Lipson, Opt. Express 15, 3140 (2007); T. Asano, W. Kunishi, M. Nakamura, B. S. Song, and S. Noda, Electron. Lett. 41, 37 (2005).

${ }^{8}$ See Refs. 9-12 and, for example, G. H. Vander Rhodes, M. S. Ünlü, B. B. Goldberg, J. M. Pomeroy, and T. F. Krauss, IEE Proc.: Optoelectron. 145, 379 (1998); S. I. Bozhevolnyi, V. S. Volkov, T. Sondergaard, A. Boltasseva, P. I. Borel, and M. Kristensen, Phys. Rev. B 66, 235204 (2002); K. Okamoto, M. Loncar, T. Yoshie, A. Sherer, Y. Qiu, and P. Gogna, Appl. Phys. Lett. 82, 1676 (2003); H. Gersen, T. J. Karle, R. J. Engelen, W. Bogaerts, J. P. Korterik, N. F. van Hulst, T. F. Krauss, and L. Kuipers, Phys. Rev. Lett. 94, 073903 (2005); M. Abashin, P. Tortora, I. Märki, U. Levy, W. Nakagawa, L. Vaccaro, H. Herzig, and Y. Fainman, Opt. Express 14, 1643 (2006).

${ }^{9}$ N. Louvion, D. Gérard, J. Mouette, F. de Fornel, C. Seassal, X. Letartre, A. Rahmani, and S. Callard, Phys. Rev. Lett. 94, 113907 (2005).

${ }^{10}$ P. Kramper, M. Kafesaki, C. M. Soukoulis, A. Birner, F. Müller, U. Gösele, R. B. Wehrspohn, J. Mlynek, and V. Sandoghdar, Opt. Lett. 29, 174 (2004).

${ }^{11}$ B. Cluzel, E. Picard, T. Charvolin, E. Hadji, L. Lalouat, F. de Fornel, C. Sauvan, and P. Lalanne, Appl. Phys. Lett. 88, 051112 (2006).

${ }^{12}$ L. Lalouat, B. Cluzel, P. Velha, E. Picard, D. Peyrade, J. P. Hugonin, P. Lalanne, E. Hadji, and F. de Fornel, Phys. Rev. B 76, 041102 (2007).

${ }^{13}$ W. C. L. Hopman, A. J. F. Hollink, R. M. de Ridder, K. O. van der Werf, V. Subramaniam, and W. Bogaerts, Opt. Express 14, 8745 (2006).

${ }^{14}$ I. Märki, M. Salt, and H. P. Herzig, Opt. Express 14, 2969 (2006).

${ }^{15}$ J. T. Robinson, S. F. Preble, and M. Lipson, Opt. Express 14, 10588 (2006).

${ }^{16}$ C. Sauvan, G. Lecamp, P. Lalanne, and J. P. Hugonin, Opt. Express 13, 245 (2005)

${ }^{17}$ A. R. M. Zain, M. Gnan, M. H. Chong, M. Sorel, and R. M. De La Rue, IEEE Photonics Technol. Lett. 20, 6 (2008).

${ }^{18}$ P. Velha, E. Picard, T. Charvolin, E. Hadji, J. C. Rodier, P. Lalanne, and D. Peyrade, Opt. Express 15, 16090 (2007).

${ }^{19}$ J. W. P. Hsu, M. Lee, and B. S. Deaver, Rev. Sci. Instrum. 66, 3177 (1995).

${ }^{20}$ A. F. Koenderink, M. Kafesaki, B. C. Buchler, and V. Sandoghdar, Phys. Rev. Lett. 95, 153904 (2005).

${ }^{21}$ J. T. Robinson, S. F. Preble, and M. Lipson, Opt. Express 14, 10588 (2006).

${ }^{22}$ E. Silberstein, P. Lalanne, J. P. Hugonin, and Q. Cao, J. Opt. Soc. Am. A 18, 2865 (2001)

${ }^{23}$ M. L. M. Balistreri, J. P. Korterik, L. Kuipers, and N. F. van Hulst, Phys. Rev. Lett. 85, 294 (2000).

${ }^{24}$ The authors notice a relevant recent publication in this field complementary to the present work: S. Mujumdar, A. F. Koenderink, T. Sünner, B. C. Buchler, M. Kamp, A. Forchel, and V. Sandoghdar, Opt. Express 15, 17214 (2007). 